\title{
RESEARCH
}

Open Access

\section{Caveolin-1 mediates soft scaffold-enhanced adipogenesis of human mesenchymal stem cells}

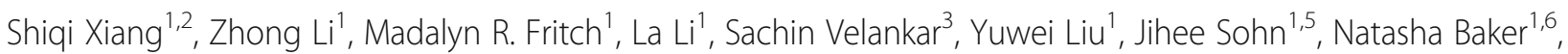
Hang $\operatorname{Lin}^{1,4,7^{*}}$ and Rocky S. Tuan ${ }^{1,4,7,8^{*}}$

\begin{abstract}
Background: Human bone marrow-derived mesenchymal stem cells (hBMSCs) can differentiate into adipocytes upon stimulation and are considered an appropriate cell source for adipose tissue engineering. In addition to biochemical cues, the stiffness of a substrate that cells attach to has also been shown to affect hBMSC differentiation potential. Of note, most current studies are conducted on monolayer cultures which do not directly inform adipose tissue engineering, where 3-dimensional (3D) scaffolds are often used to create proper tissue architecture. In this study, we aim to examine the adipogenic differentiation of hBMSCs within soft or stiff scaffolds and investigate the molecular mechanism mediating the response of hBMSCs to substrate stiffness in 3D culture, specifically the involvement of the integral membrane protein, caveolin-1 (CAV1), known to regulate signaling in MSCs via compartmentalizing and concentrating signaling molecules.
\end{abstract}

Methods: By adjusting the photo-illumination time, photocrosslinkable gelatin scaffolds with the same polymer concentration but different stiffnesses were created. hBMSCs were seeded within soft and stiff scaffolds, and their response to adipogenic induction under different substrate mechanical conditions was characterized. The functional involvement of CAV1 was assessed by suppressing its expression level using CAV1-specific siRNA.

Results: The soft and stiff scaffolds used in this study had a compressive modulus of $\sim 0.5 \mathrm{kPa}$ and $\sim 23.5 \mathrm{kPa}$, respectively. hBMSCs showed high viability in both scaffold types, but only spread out in the soft scaffolds. hBMSCs cultured in soft scaffolds displayed significantly higher adipogenesis, as revealed by histology, qRT-PCR, and immunostaining. Interestingly, a lower CAV1 level was observed in hBMSCs in the soft scaffolds, concomitantly accompanied by increased levels of Yes-associated protein (YAP) and decreased YAP phosphorylation, when compared to cells seeded in the stiff scaffolds. Interestingly, reducing CAV1 expression with siRNA was shown to further enhance hBMSC adipogenesis, which may function through activation of the YAP signaling pathway.

Conclusions: Soft biomaterials support superior adipogenesis of encapsulated hBMSCs in 3D culture, which is partially mediated by the CAV1-YAP axis. Suppressing CAV1 expression levels represents a robust method in the promotion of hBMSC adipogenesis.

Keywords: Mesenchymal stem cells, Substrate stiffness, Photocrosslinked gelatin hydrogel, Adipogenesis, YAP, CAV1

\footnotetext{
*Correspondence: hal46@pitt.edu; tuanr@cuhk.edu.hk

${ }^{1}$ Center for Cellular and Molecular Engineering, Department of Orthopaedic

Surgery, University of Pittsburgh School of Medicine, Pittsburgh,

Pennsylvania, USA

Full list of author information is available at the end of the article
}

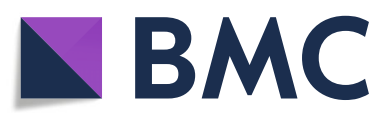

(- The Author(s). 2021 Open Access This article is licensed under a Creative Commons Attribution 4.0 International License, which permits use, sharing, adaptation, distribution and reproduction in any medium or format, as long as you give appropriate credit to the original author(s) and the source, provide a link to the Creative Commons licence, and indicate if changes were made. The images or other third party material in this article are included in the article's Creative Commons licence, unless indicated otherwise in a credit line to the material. If material is not included in the article's Creative Commons licence and your intended use is not permitted by statutory regulation or exceeds the permitted use, you will need to obtain permission directly from the copyright holder. To view a copy of this licence, visit http://creativecommons.org/licenses/by/4.0/. The Creative Commons Public Domain Dedication waiver (http://creativecommons.org/publicdomain/zero/1.0/) applies to the data made available in this article, unless otherwise stated in a credit line to the data. 


\section{Background}

Fat is the most abundant tissue of the human body. It provides structural protection, shapes a normal human appearance, and plays key roles in endocrine and metabolic functions [1]. Due to aging, trauma, or pathological conditions, one can suffer damage to or significant losses in adipose tissue, often requiring surgical interventions. Free-fat transplantation represents a classic approach for replacing lost or damaged adipose tissue, but is often limited by delayed neovascularization, graft volume shrinkage, and poor long-term survival [2]. In recent years, the emerging technologies of tissue engineering strategy, which typically involve the generation of new tissues through guided differentiation of cells encapsulated a 3-dimenstional (3D) scaffold, have presented alternative approaches for regenerating adipose tissue. Specifically, adult mesenchymal stem cells derived from the human bone marrow (hBMSCs) or fat, which have shown robust adipogenic potential upon stimulation, are the two most used cell sources in current adipose tissue engineering [3-5]. To generate tissues with sufficient volume for large defect repair, these cells are often combined with scaffolds that provide structural support as well as influence cell behavior through cell-matrix interactions. For example, robust adipogenesis was reported for hBMSCs seeded with gelatin-based scaffolds [6] and polylactic acid nanofibrous scaffold [7] upon treatment with adipogenic medium.

In addition to the inducing agents present in adipogenic medium, hBMSC adipogenesis is also affected by biomechanical signals [8-14]. Majumder et al. [15] reported that a soft substrate is better able to maintain the adipogenic differentiation ability of hBMSCs. Similar results were reported in another study, which demonstrated that a greater degree of adipogenesis occurs on softer matrices, as evidenced by the accumulation of lipid droplets [16]. Of note, most current studies investigating the response of hBMSCs to substrate stiffness were conducted on two-dimensional (2D) cultures [17]. Although this conventional culture condition allows easy cell manipulation and analysis, it does not assess 3D cultures commonly used in tissue engineering. Therefore, an increasing number of studies have recently been focusing on cell behavior in 3D culture environments. For example, Chaudhuri et al. seeded hBMSCs in alginate scaffolds and found that cells cultured in a softer alginate scaffold produced more oil drops and expressed higher levels of adipogenic genes than cells in stiff scaffolds [18]. Based on current studies, it is generally accepted that softer substrates enhance the level of adipogenesis, irrespective of 2D or 3D culture [19].

Integrins and associated molecules, specifically the YAP (Yes-associated protein) and its paralog TAZ (transcriptional co-activator with PDZ-binding motif), components of the Hippo signaling cascade, have been shown to play a role in the response of hBMSCs to substrate stiffness [20]. However, the outcome of differentiation also depends highly on the dimensionality of the culture system [21]. Interestingly, integrin endocytosis, one means of integrin regulation, is mediated by caveolin-1 (CAV1) coated-caveolae membrane rafts [22]. It has also been reported that CAV1 positively modulates YAP activity under 2D conditions [23]. To date, the interplay between CAV1 and YAP/TAZ during the cell's response to substrate stiffness and adipogenic medium in the context of $3 \mathrm{D}$ culture conditions has not been fully studied.

Herein, we aim to examine the efficiency of hBMSC adipogenesis within soft and stiff hydrogels and investigate how CAV1 and YAP/TAZ regulate the response of hBMSCs to substrate stiffness. We aimed to test the hypothesis that a soft 3D scaffold would support a higher level of adipogenesis than a stiff one. To test this hypothesis, we first prepared photo-crosslinked hydrogels with the same gelatin concentrations but different stiffnesses by adjusting the time the scaffolds were cured under photo-crosslinking illumination. Then, the viability and morphology of hBMSCs within soft and stiff scaffolds were examined. After hBMSC-laden soft or stiff scaffolds were cultured in adipogenic medium for 2 weeks, the level of adipogenesis was examined by qRTPCR, histology, and immunostaining. Lastly, we assessed the alterations of CAV1 and YAP levels in cells encapsulated in soft and stiff scaffolds during adipogenesis and then used siRNA to downregulate the expression levels of CAV1 to examine the role of CAV1 and YAP in regulating adipogenesis in the context of 3D culture.

\section{Methods}

\section{Preparation of hydrogel scaffolds with different levels of stiffness}

Gelatin scaffolds were fabricated using previously reported methods [24, 25]. Briefly, to synthesize the methacrylated gelatin (GelMA), bovine skin-derived gelatin (Sigma-Aldrich, St. Louis, MO) was dissolved in deionized $\mathrm{H}_{2} \mathrm{O}$ and then reacted with methacrylic anhydride (Sigma-Aldrich) overnight at $37^{\circ} \mathrm{C}$. The reaction product was dialyzed against distilled water using a dialysis cassette $(3.5 \mathrm{~K}$ molecular weight cut-off membrane, ThermoFisher, Waltham, MA) for 5 days to remove the salts and methacrylic acid. The solution was then lyophilized, and the dried sponge was stored in a desiccator. To make the monomer solution, GelMA was dissolved into Hanks' Balanced Salt Solution (HBSS, with $\mathrm{Ca}^{2+}$ and $\mathrm{Mg}^{2+}$; ThermoFisher) at $15 \%(\mathrm{w} / \mathrm{v})$, and the photoinitiator, lithium phenyl-2,4,6-trimethyl-benzoyl phosphinate (LAP, Sigma-Aldrich, St. Louis, MO), was added at $0.15 \%(\mathrm{w} / \mathrm{v})$. 
To generate the hydrogel scaffolds, the GelMA/LAP solution was first poured into silicon molds, which had a cylindrical void space $(2 \mathrm{~mm}$ height $\times 5 \mathrm{~mm}$ diameter). Then, polymerization was induced using a flashlight with a wavelength at $395 \mathrm{~nm}$ for $8 \mathrm{~s}$ or 2 min to generate soft or stiff scaffolds.

\section{Degradation behavior of hydrogels}

The biodegradability of hydrogel scaffolds was evaluated by incubating gels in collagenase type 1 solution in phosphate-buffered saline (PBS) $(0.05 \%, \mathrm{w} / \mathrm{v})$ (Worthington-Biochemical Corporation, Lakewood, $\mathrm{NJ}$ ) at $37^{\circ} \mathrm{C}$ with shaking at $50 \mathrm{rpm} / \mathrm{min}$. At different time points, samples were taken, washed, and weighed. Biodegradability was quantified by determining the $\%$ remaining weight as follows:

$$
\% \text { Weight remaining }=\mathrm{W}_{\mathrm{t}} / \mathrm{W}_{0} \times 100 \% \text {. }
$$

where $\mathrm{W}_{0}$ was the initial wet weight of the hydrogel scaffold and $W_{t}$ is the weight at different time points $(t)$.

\section{Mechanical test}

The compressive moduli of hydrogel scaffolds created with different photo-illumination times were measured using a mechanical tester (Bose ElectroForce 3230 Series II, TA Instruments, New Castle, DE). Briefly, the hydrogels were subjected to $10 \%$ compression $(0.2 \mathrm{~mm})$ at $0.01 \mathrm{~mm} / \mathrm{s}$, and the linear portion of the stress-strain curve was used to calculate the compressive modulus of the scaffolds.

\section{Rheological analysis}

The continuous and oscillatory shear measurements at small strain were used to characterize the viscoelastic properties of the hydrogel scaffolds. Both experiments were conducted using an Anton Paar MCR 302 rheometer (Ashland, VA). For test preparation, the scaffolds were placed between two profiled parallel plates with a diameter of $25 \mathrm{~mm}$ to prevent wall slip and were preheated at $37{ }^{\circ} \mathrm{C}$ to simulate body temperature. A preshear test was first run at a shear rate of $1 \mathrm{~s}^{-1}$ for 10 min for each sample. The shear rates for the continuous shear tests increased from 0.1 to $100 \mathrm{~s}^{-1}$, with a $1 \mathrm{~min}$ hold at each shear rate. In the oscillatory experiments, amplitude sweep tests were carried out with the strain ranging from $0.01 \%$ up to $100 \%$ at a constant frequency of $1 \mathrm{~s}^{-1}$.

\section{Harvest and expansion of human bone marrow-derived mesenchymal stem cells (hBMSCs)}

According to an Institutional Review Board (IRB) exempted approval protocol (University of Washington), the surgical waste from total joint replacements was used for hBMSC isolation. The methods have been reported in our previous studies [24, 26]. Briefly, the femoral heads were flushed with a rinsing medium ( $\alpha$-MEM and $1 \%$ antibiotic-antimycotic, Invitrogen, Carlsbad, CA). The cell suspension was centrifugated, and the pellet was resuspended in the growth medium [GM, Dulbecco's modified Eagle's medium (DMEM; Gibco, Grand Island, NY) supplemented with $10 \%$ (v/v) fetal bovine serum (FBS, Gemini Bio-Products, West Sacramento, CA) and $1 \times$ antibiotic-antimycotic (anti-anti; Gibco)] supplemented with $1.5 \mathrm{ng} / \mathrm{mL}$ fibroblast growth factor-2 (FGF-2; RayBiotech, Norcross, GA) and cultured in T150 flasks (Corning Inc., corning, NY)). Upon reaching 70-80\% confluence, cells were detached by trypsin$0.25 \%$ ethylenediaminetetraacetic acid (ThermoFisher, Waltham, MA) and passaged. At passage 3 (P3), hBMSCs isolated from 5 male and 5 female donors were pooled. P5 cells were used in all experiments.

\section{In vitro culture of cell-laden gelatin scaffolds}

To generate the two cell-laden hydrogel scaffolds with different stiffnesses, hBMSC pellets were resuspended in GelMA/LAP solution at a final density of $10 \times 10^{6}$ cells/ $\mathrm{ml}$ and then subjected to $8 \mathrm{~s}$ (soft) or $2 \mathrm{~min}$ (stiff) illumination using the method described above. The hBMSC-laden soft and stiff hydrogels were cultured in GM overnight and then maintained in adipogenic medium [27] (AM: $\alpha$-MEM (Gibco) supplemented with $10 \%$ FBS, 1\% antibiotics-antimycotics, $0.45 \mathrm{mM} \mathrm{3-}$ isobutyl-1-methylxanthine (Sigma-Aldrich, St. Louis, $\mathrm{MO}), 0.1 \mu \mathrm{M}$ dexamethasone (Sigma-Aldrich), $0.2 \mathrm{mM}$ indomethacin (Sigma-Aldrich), and $1 \times$ insulintransferrin-selenium (ITS, Invitrogen)).

\section{Analysis of cell morphology and cell viability in 3D hydrogels}

Both cell morphology and cell viability were assessed after 1, 4, and 7 days of culture in AM. For morphological observation, the cell-laden scaffolds were fixed in $4 \%$ paraformaldehyde (PFA) aqueous solution (Fisher Scientific, Hampton, NH) for $2 \mathrm{~h}$ and permeabilized with 0.1\% Triton X-100 (Sigma-Aldrich). Alexa Fluor 488 phalloidin (Invitrogen, Carlsbad, CA) was used to label cytoskeletal actin filaments, and Hoechst 33342 solution (Invitrogen, Carlsbad, CA) was utilized as nuclear counterstain. To assess cell viability, the LIVE/DEAD cell viability assay (Life Technologies, Carlsbad, CA) was used. Briefly, constructs were transferred into maintenance medium (phenol red-free DMEM (Gibco) containing $10 \%(\mathrm{v} / \mathrm{v}) \mathrm{FBS}$ ), containing calcein $\mathrm{AM}$ and ethidium homodimer-1, and then incubated at $37^{\circ} \mathrm{C}$ for $30 \mathrm{~min}$. Images were acquired using an Olympus Fluoview 1000 confocal microscope (Center Valley, PA). $Z$ stacks were acquired at optimal intervals $(2 \mu \mathrm{m}$ or $4 \mu \mathrm{m}$ steps, 100 
$150 \mu \mathrm{m}$ stack) as suggested by the software. NIH ImageJ software was utilized to analyze all the confocal stacks.

\section{Gene expression analysis}

To isolate total cellular RNA, cell-laden scaffolds were transferred into a 1.5-ml RNase-free microtube and cells were lysed in QIAzol reagent, followed by RNA extraction using a RNeasy Plus Universal Kit (Qiagen, Germantown, MD). Reverse transcription was then carried out using the SuperScript ${ }^{\circ}$ VILO $^{\mathrm{TM}}$ cDNA Synthesis Kit (Invitrogen) to obtain complementary DNA. qRT-PCR was then performed using SYBR Green chemistry and the QuantStudio 3 qRT-PCR system (Applied Biosystems, Foster City, CA). Relative gene expression was calculated using the comparative $\mathrm{Ct}\left(2^{-\Delta \Delta \mathrm{CT}}\right)$ method, and the housekeeping gene glyceraldehyde-3-phosphatase dehydrogenase $(G A P D H)$ was used as the endogenous control. The sequences of primers are listed in Table S1.

\section{Histology and immunostaining}

The cell-laden 3D hydrogel constructs were fixed in 4\% PFA and embedded in Cryo-gel (Leica Microsystems Inc, Chicago, IL). Blocks were sectioned at $6-\mu \mathrm{m}$ thickness, and Oil Red $\mathrm{O}$ staining was performed to detect the lipid droplet [27]. The stained sections or cultures in 6-well plates were imaged with an EVOS M5000 Imaging System (ThermoFisher, Waltham, MA). To quantify the lipid content, cells on the culture plates were first washed exhaustively in distilled water and dried by placing it at $32{ }^{\circ} \mathrm{C}$ for $40 \mathrm{~min}$ [28]. Isopropyl alcohol was added to elute the dye from the cells and dye intensity was estimated spectrophotometrically based on $\mathrm{A}_{510}$ (Microplate Reader, BioTek, Winooski, VT).

BODIPY 493/503 (10 $\mu \mathrm{g} / \mathrm{ml}$, Invitrogen, D3922) was also used for lipid detection. Cryosections were washed with $1 \%(\mathrm{v} / \mathrm{v})$ PBS-T solution for $10 \mathrm{~min}$ and then incubated with BODIPY solution for $30 \mathrm{~min}$. Hoechst 33342 (ThermoFisher, H3570) was used as nuclear counterstain.

For immunofluorescence (IF), the 4\% PFA-fixed cryosections were first blocked in 10\% goat serum (ab7481, Abcam, Cambridge, UK) in PBS for $1 \mathrm{~h}$ and incubated with primary antibodies against C/EBP- $\alpha$ (8178s, Cell Signaling Technology, Danvers, MA, 1:100 dilution) or CAV1 (3238s, Cell Signaling Technology, 1:400 dilution) overnight at $4^{\circ} \mathrm{C}$. The information on the primary antibodies used in this study is listed in Table S2. For secondary antibodies, a goat anti-rabbit IgG (Alexa Fluor 488; Abcam, 1:500 dilution) was utilized. Alexa Fluor 568 conjugated phalloidin (Invitrogen, A12380, 1:200 dilution) was used to stain actin fibers. The samples were then mounted with a 4',6-diamidino-2-phenylindole (DAPI)-containing antifade medium (Vector Laboratories, Burlingame, CA). An Olympus IX81 inverted microscope (Olympus, Waltham, MA) and EVOS M5000
Imaging System (ThermoFisher) were used to image the stained sections.

\section{Western blot analysis}

Constructs were washed in PBS for 3 times and then pulverized in RIPA buffer (Sigma Aldrich) supplemented with $1 \%(\mathrm{v} / \mathrm{v})$ protease and phosphatase inhibitor cocktail (ThermoFisher). After centrifugation, protein concentrations of the supernatants were determined using the BCA protein assay kit (Thermo Scientific BCA Protein Assay Kit). After thermal denaturation and reduction in Laemmli buffer containing $\beta$-mercaptoethanol $(10 \%(\mathrm{v} / \mathrm{v})$ (BioRad; Hercules, California), the proteins were electrophoretically fractionated in a $4-12 \%$ Bis-Tris polyacrylamide gel (Invitrogen ${ }^{\mathrm{Tx}}$, NP0326BOX) and transferred onto PVDF membranes $(0.2 \mu \mathrm{m})$. After being blocked in $3 \%$ non-fat milk (BioRad) in TBST $(0.1 \%$ Tween-20 in TBS) for $1.5 \mathrm{~h}$ at room temperature, the membranes were incubated at $4^{\circ} \mathrm{C}$ overnight with primary antibodies against target proteins (diluted in 1\% non-fat milk in TBST; see information in Table S2). After washing in TBST for 5 times, the membranes were incubated with horseradich peroxidase (HRP)-conjugated secondary antibodies (GENA934-1ML, Sigma-Aldrich, 1:2000 dilution) for $1.5 \mathrm{~h}$ at room temperature and then with SuperSignal West Femto Maximum Sensitivity Substrate solution (ThermoFisher). The blots were imaged using the ChemiDocTM Touch Imaging System (Bio-Rad). Quantification of the blot images was conducted using NIH ImageJ.

\section{siRNA treatment}

siRNA transfection was performed as described previously [29]. Briefly, small interfering RNA (CAV1, Invitrogen) and negative control siRNA (Silencer Negative Control No. 1 siRNA, Invitrogen) were incubated with Lipofectamine $^{\text {nx }}$ RNAiMax reagent (Invitrogen) respectively in Opti-MEM medium for 5-10 min. The mixture was added to cell culture and incubated at $37^{\circ} \mathrm{C}$ for $48 \mathrm{~h}$. Transfection efficiency was assessed using Western blot and qRT-PCR. The transfected cells were subjected to $3 \mathrm{D}$ culture using the same conditions described above.

\section{Statistical analyses}

Statistical analysis was carried out using GraphPad Prism 9 (GraphPad, San Diego, CA). All data are presented as means and 95\% confidence intervals for analyzing the correlation of gene expression. Mean differences between the two groups were assessed with Student's $t$ test. Analysis of variance (ANOVA) was used to analyze results among multiple groups. $p$ values less than 0.05 were considered statistically significant, and depicted in figures as ${ }^{*} p<0.05,{ }^{* * *} p<0.01,{ }^{* * * * *} p<0.001$, and ${ }^{* * * * *} p<$ 0.0001 . 


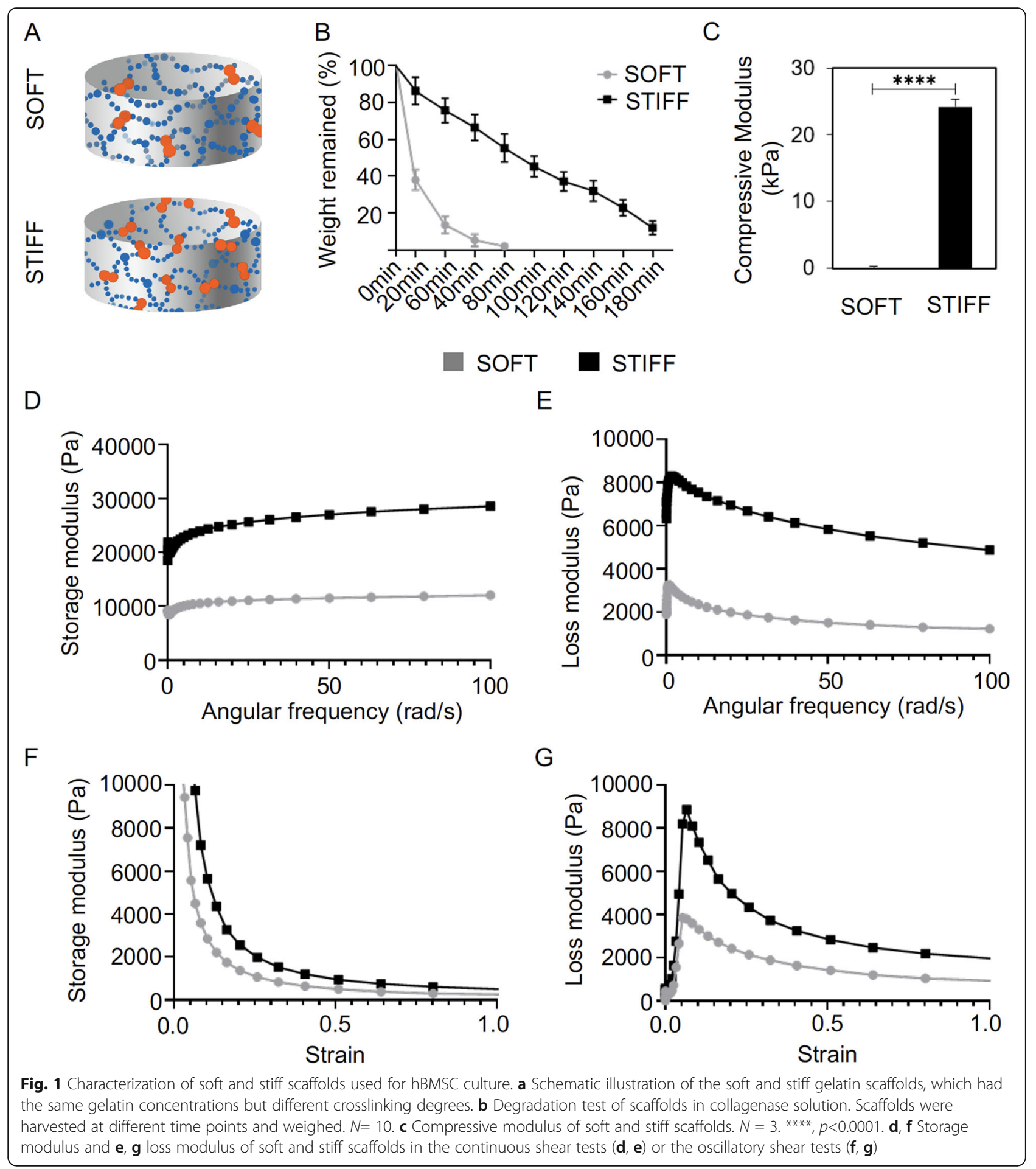

\section{Results}

Soft and stiff scaffolds are fabricated from GelMA with a same concentration

In this study, GelMA was used to fabricate scaffolds with different stiffnesses. The degree of crosslinking was controlled by adjusting the light exposure time ( 8 or $120 \mathrm{~s}$ ), to create soft and stiff scaffolds, respectively (Fig. 1a). In both scaffolds, the gelatin concentration was the same $(15 \% \mathrm{w} / \mathrm{v})$. Upon collagenase treatment, soft scaffolds displayed faster degradation rates. Thus, in less than 80 min, the soft scaffolds were completely degraded (Fig. 1b). In contrast, it took more than $180 \mathrm{~min}$ to degrade stiff scaffolds. As expected, soft scaffolds had a significantly lower average compressive modulus of $0.5 \mathrm{kPa}$, 


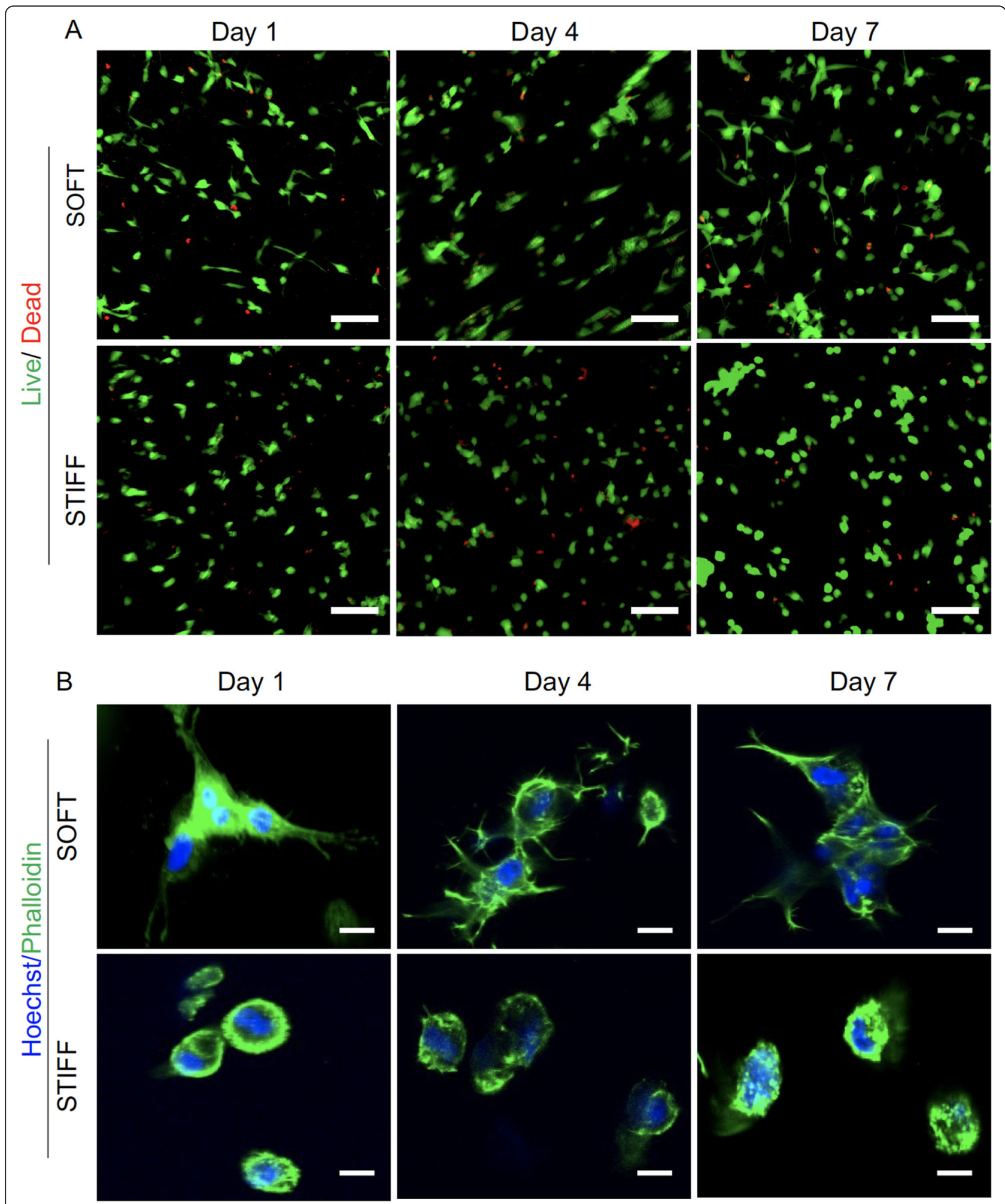

Fig. 2 Viability and morphology of hBMSCs cultured in soft and stiff scaffolds. a Live/Dead staining of hBMSCs cultured for 1, 4, and 7 days. Green $=$ live cells; Red $=$ dead cells; Bar $=100 \mu \mathrm{m}$. b Representative higher magnification imaging by confocal microscopy showing cell morphology. $\operatorname{Bar}=20 \mu \mathrm{m}$ 


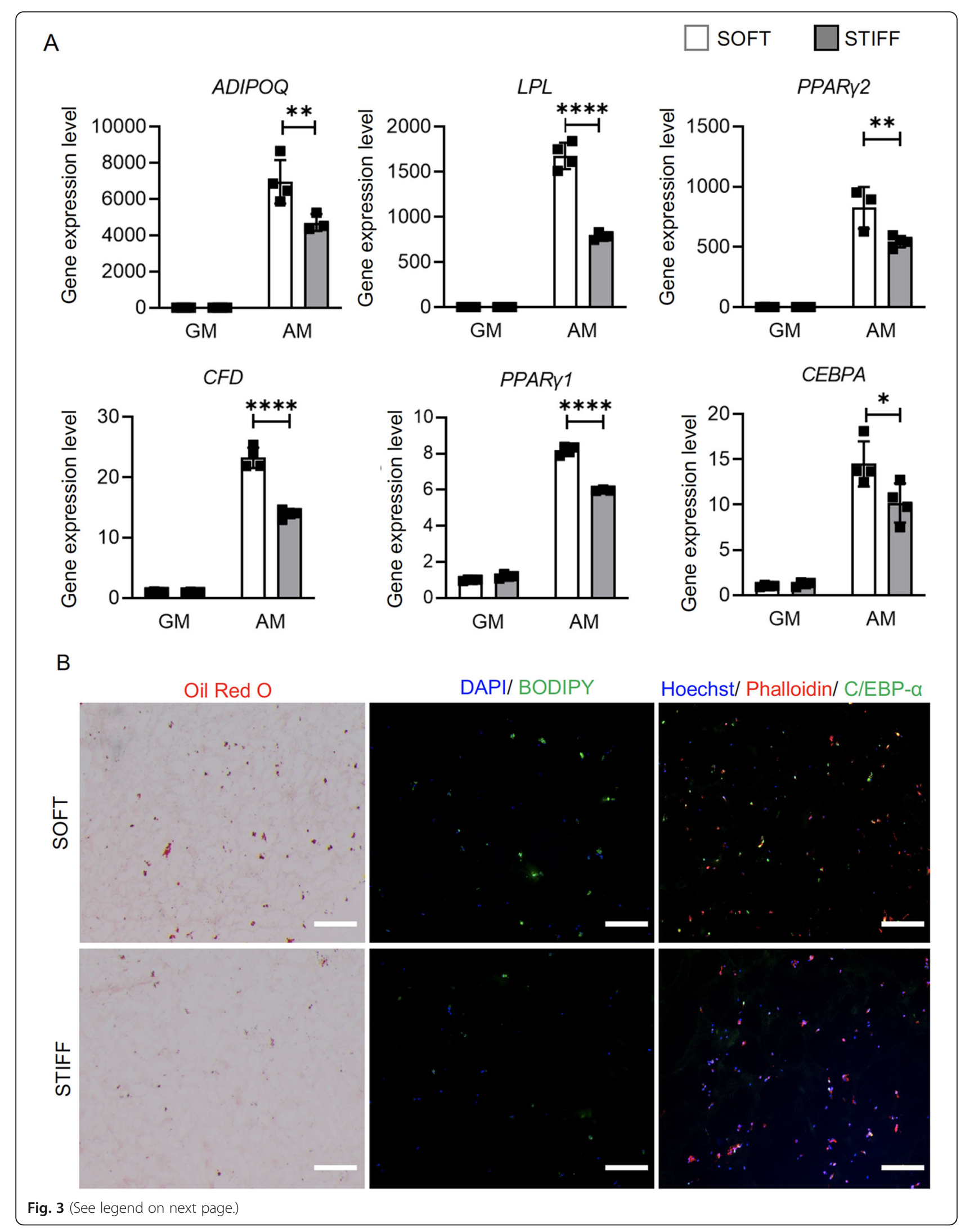


(See figure on previous page.)

Fig. 3 Assessment of adipogenesis in hBMSC-laden scaffolds. a Relative expression levels of adipogenic marker genes in hBMSCs cultured under adipogenic conditions (AM) in soft or stiff scaffolds. Data are normalized to that from the Soft-GM group (cultured under control growth conditions). $N=4 .{ }^{*} p<0.05$, ${ }^{* *} p<$ $0.01,{ }^{* * * *} p<0.0001$. GM: growth medium, AM: adipogenic medium. b Oil Red O staining, BODIPY staining, and C/EBP-a immunofluorescence to assess adipogenesis. Bar $=100 \mu \mathrm{m}$

while stiff scaffolds had an average compressive modulus of $23.5 \mathrm{kPa}$ (Fig. 1c).

Figures 1d, e shows that in the continuous shear tests, as the crosslinking time increased from $8 \mathrm{~s}$ to $120 \mathrm{~s}$, the storage and loss moduli of the hydrogel both significantly increased in the frequency range of $0.1-100 \mathrm{~s}^{-1}$. The storage and loss moduli values relate to the hydrogel's elastic and viscous behaviors, respectively. For both sample groups, the storage moduli values were substantially larger than the loss storage values, indicating that soft and stiff hydrogels both displayed mainly elastic behavior. In the oscillatory shear tests (Fig. 1f, g), larger storage and loss moduli values were also observed for the stiff hydrogels when strains of 0.01 to $100 \%$ were applied, suggesting a larger resistance to deformation caused by forces exerted by the encapsulated cells. Therefore, cells gown in the stiff hydrogels were expected to experience a larger constraint by the local matrix than those in the soft hydrogels. In addition, both soft and stiff hydrogels showed a drastic decrease in storage moduli values in the strain range of $0.01-20 \%$, indicating their decreased ability to recover from larger deformations.

\section{hBMSCs display different morphologies when cultured in soft and stiff scaffolds}

hBMSCs encapsulated in both soft and stiff scaffolds were assessed for cell viability and morphology on days 1, 4, and 7. As shown in Fig. 2a, hBMSCs displayed high viability $(>90 \%)$ in both scaffolds at all time points tested. Interestingly, phallodin cytoskeletal staining showed that cells within soft scaffolds spread and extended in all directions (Fig. 2b, Video S1), whereas cells within stiff scaffolds displayed a rounder morphology with limited development of actin filaments (Fig. 2b, Video S2).

\section{Culturing within soft scaffolds results in higher level of hBMSC adipogenesis}

hBMSC-laden soft and hard scaffolds were cultured in adipogenic medium for 14 days. As shown in Fig. 3a, all tested adipogenic genes were upregulated upon adipogenic stimulation. When compared to those in stiff scaffolds, cells within soft scaffolds showed higher expression levels of adipogenic genes, as well as produced more histologically detectable lipid droplets, $\mathrm{C} / \mathrm{EBP}-\alpha$ protein and BODIPY staining (Fig. 3b). A similar trend was observed on day 7 as well (Figure S1), which collectively implied a higher level of adipogenesis in soft scaffolds.

\section{hBMSCs cultured in soft scaffolds express lower level of CAV1}

We then used immunofluorescence and Western blot to examine the expression level of CAV1. As shown in Fig. 4a, d, lower CAV1 levels were observed in softer scaffolds than in stiff scaffolds at all tested time points, except on day 1 . In addition, as shown in Fig. 4b, c, higher levels of YAP and lower levels of YAP phosphorylation ( $\mathrm{p}$-YAP:YAP) were observed in cultures maintained in the soft scaffolds, suggesting that YAP activation is higher in soft cultures. In summary, CAV1 and YAP are closely related in regulating cellular responses to stiffness and adipogenic stimulation.

\section{Knockdown of $C A V-1$ results in enhanced adipogenesis}

To understand the function of CAV1, siRNA was used to reduce the expression levels of CAV1. Transfection with $C A V 1$ siRNA resulted in an $\sim 8$-fold reduction in the expression levels of CAV1 $48 \mathrm{~h}$ after transfection (Figure S2). This knockdown effect lasted up to 2 weeks (Fig. 5a). When compared to the untreated control (CTRL group) or control siRNA-treated cells (si-CTRL group), hBMSCs treated with $C A V-1$ siRNA (si-CAV1 group) displayed higher levels of adipogenesis upon adipogenic stimulation in 2D culture, revealed by qRT-PCR and Oil Red O staining (Fig. 5a, b). In particular, cells in the si-CAV1 group generated $\sim 50 \%$ more oil droplet staining than those in the si-CTRL group (Fig. 5c).

Similar CAV1 knockdown studies were conducted in 3D cultures of hBMSCs. Results from qRT-PCR, the western blot and immunofluorescence (Fig. 6a-c, e) showed that CAV1 levels were successfully reduced in 3D culture after siRNA treatment. Reducing CAV1 level resulted in increased expression of adipogenic genes (Fig. 6a), higher protein levels of PPAR- $\gamma$ (Fig. 6b, d) and C/EBP- $\alpha$ (Fig. 6e), as well as increased oil droplet deposition and BODIPY staining (Fig. 6e) than control counterparts.

\section{YAP/TAZ pathway is regulated by CAV1}

We then examined the crosstalk between CAV1 and YAP signaling during hBMSC adipogenesis. Western analysis showed that si-CAV1 transfection efficiently reduced CAV1 in cultures maintained in both soft and stiff scaffolds (Fig. 7a, b). Interestingly, in the si-CAV1 group maintained in soft scaffolds (Fig. 7a, c), protein levels of 


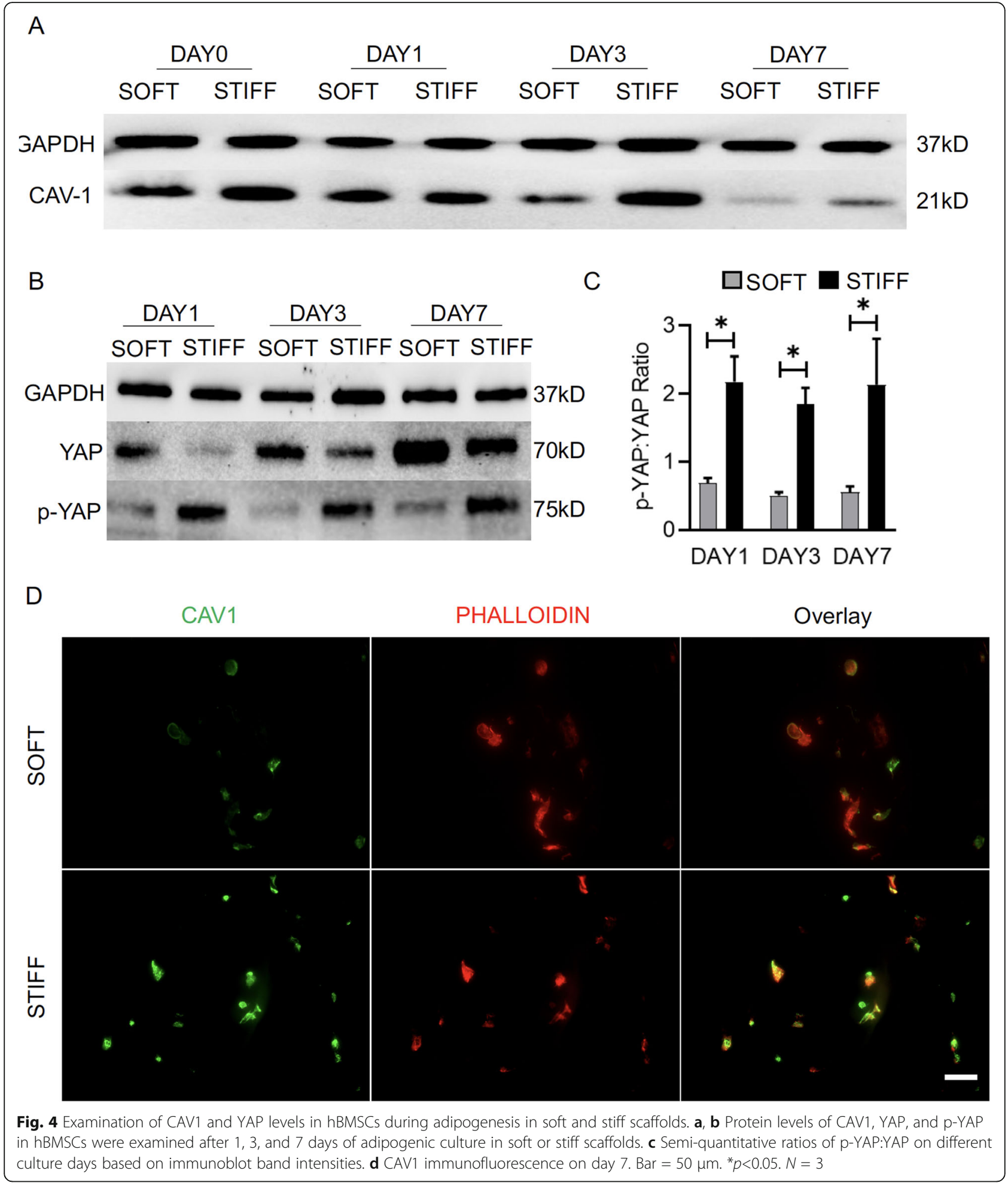

YAP and TAZ rapidly increased and YAP phosphorylation (p-YAP:YAP) decreased, while CAV1 protein levels were reduced (Fig. $7 a-c)$. In contrast, cells within stiff scaffolds displayed higher levels of CAV1, with significantly lower levels of YAP and TAZ, but higher level of
YAP phosphorylation (p-YAP:YAP); however, upon siCAV1 transfection, i.e., with reduction in CAV1 level, YAP and TAZ levels increased, and YAP phosphorylation was reduced (Fig. $7 \mathrm{a}, \mathrm{c}$ ), similar to the response to si-CAV1 treatment seen in the soft scaffold group. These 


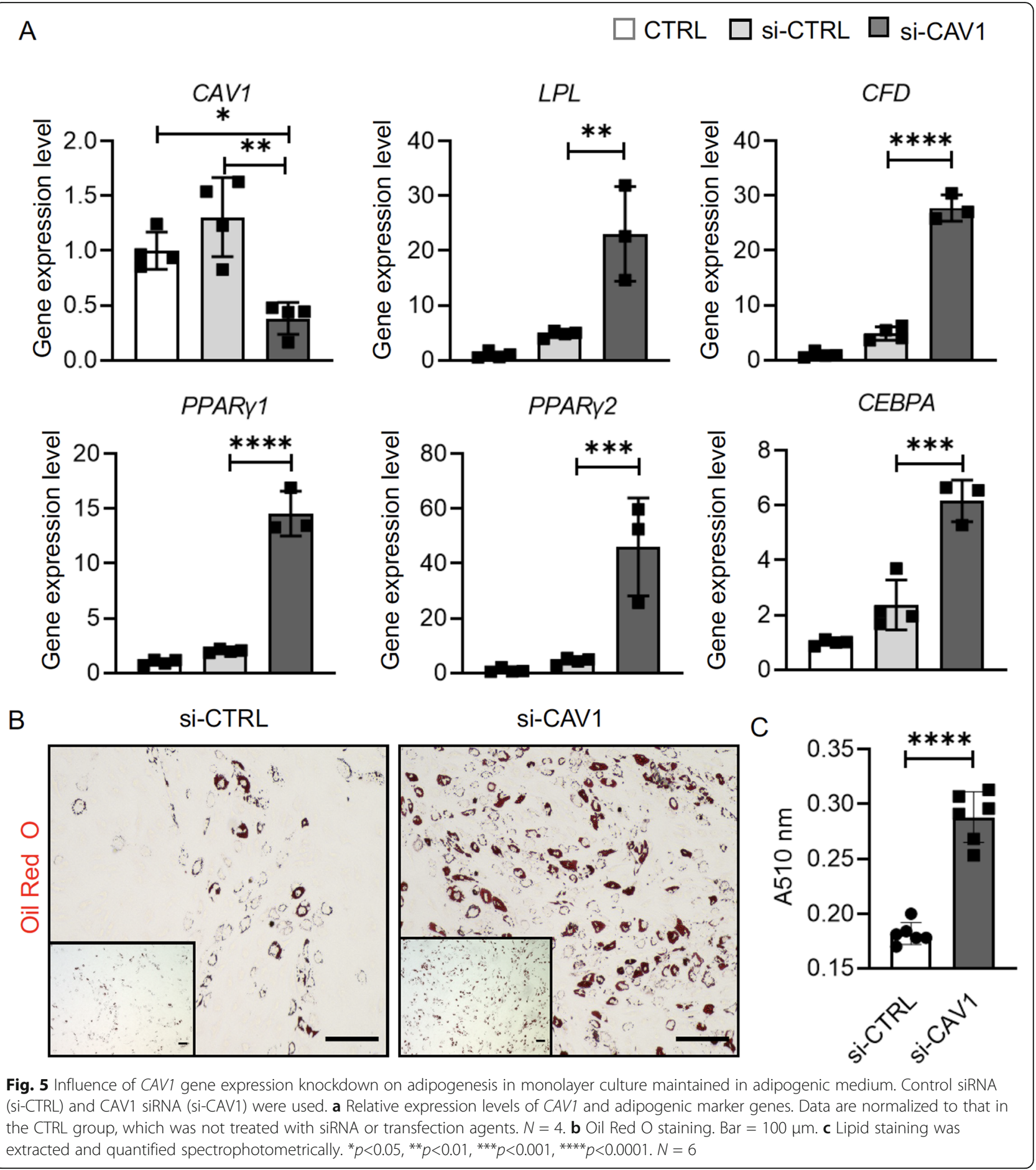

results indicated that YAP/TAZ activation, i.e., unphosphorylated versus phosphorylated YAP, was similarly affected and involved in the modulation of hBMSC adipogenesis as a function of CAV1 level.

\section{Discussion}

Adipose tissue engineering provides a robust tool to regenerate lost or damaged fat. In vitro created adipose tissue has recently been used to model diseases and develop drugs [27, 30-32]. There is thus a demonstrated need for functional fat tissues generated through tissue engineering. In this study, we have induced hBMSCs to undergo adipogenesis within 3D gelatin hydrogels and comparatively analyzed the influence of varying mechanical stiffness of the 3D scaffold in soft supporting adipogenesis. Specifically, we have studied the involvement of 


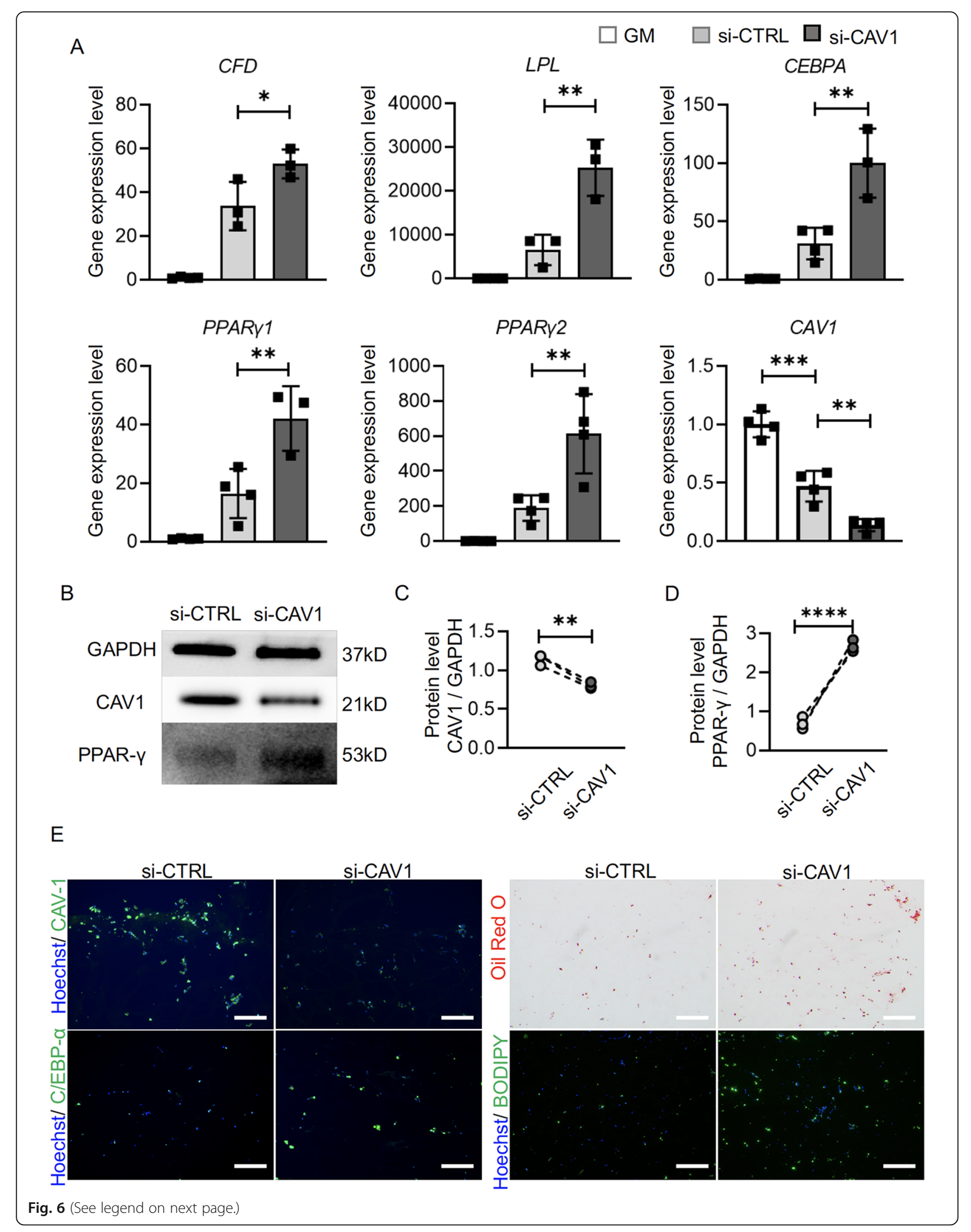


(See figure on previous page.)

Fig. 6 Influence of CAV1 gene expression knockdown on hBMSC adipogenesis in soft and stiff scaffolds. Control siRNA (si-CTRL) and CAV1 siRNA (si-CAV1) were used. a Relative expression levels of adipogenic marker genes and CAV1. Data were normalized to that in GM group. $N=4$. b, $\mathbf{c}, \mathbf{d}$ Western blot and semi-quantitative analysis of CAV1 and PPAR-y levels. $N=3$. e Immunofluorescence analysis of CAV1 and C/EBP-a levels. Bar $=$ $100 \mu \mathrm{m}$. Oil Red O and BODIPY staining of lipid deposition in cells. Bar $=100 \mu \mathrm{m} .{ }^{*} p<0.05,{ }^{* *} p<0.01,{ }^{* * *} p<0.001,{ }^{* * * *} p<0.0001$

CAV1, previously shown to regulate osteogenic differentiation of hBMSCs and the potential role of YAP/TAZ signaling in mediating cell responses to substrate stiffness and adipogenic medium in the context of 3D culture conditions.
Based on these observations, we propose how CAV1 and YAP/TAZ act to mediate hBMSC adipogenesis and responses to stiffness of 3D scaffolds (Fig. 8). Specifically, hBMSCs in soft scaffolds display low CAV1 levels, which lead to high expression levels of YAP/TAZ (with corresponding decrease in $\mathrm{p}$-YAP and thus reduced

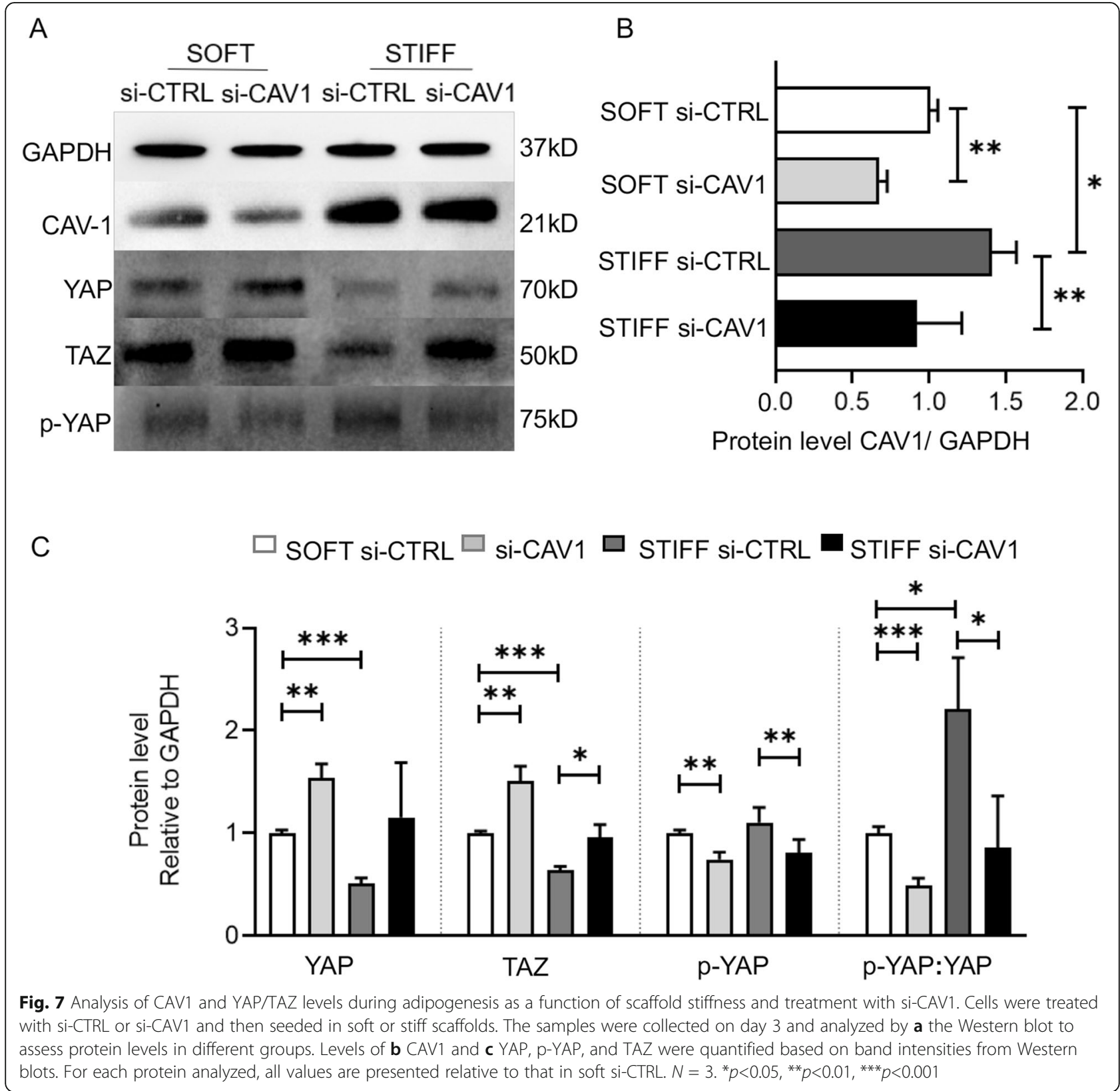




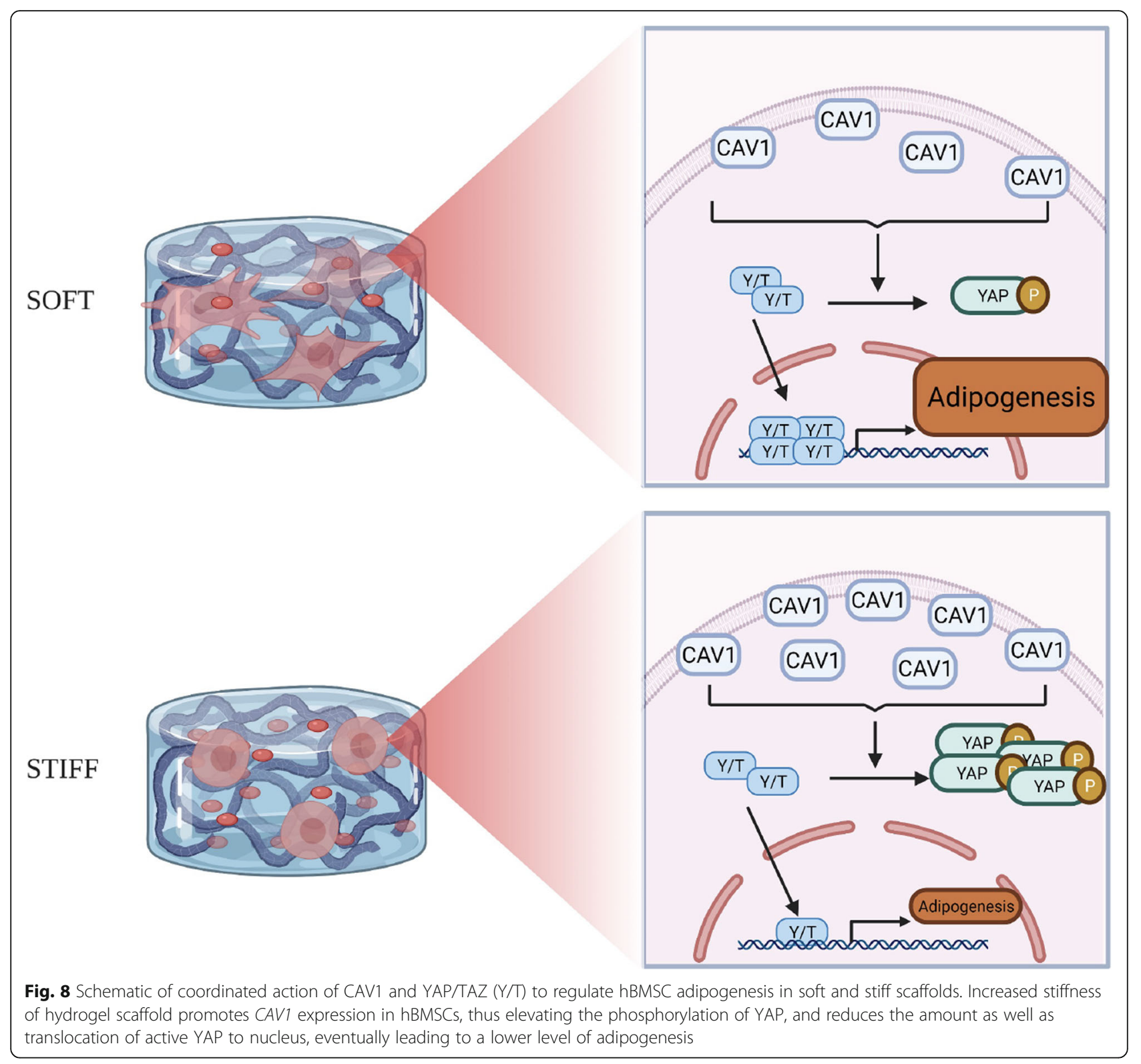

proteasomal degradation of YAP), resulting in subsequent translocation of YAP/TAZ into the nuclei, presumably activating the transcription of adipogenic genes. The si-CAV1-mediated knockdown of CAV1 further increases the activation of the YAP/TAZ pathway and adipogenesis. Conversely, cells maintained in stiff scaffolds maintain a high level of CAV1, which consequently lead to low activation of YAP/TAZ and higher level of YAP phosphorylation, resulting in reduced adipogenesis.

Given its bioactivities and biocompatibility, gelatin has been widely used in tissue engineering [33]. Its recognized biosafety makes it a convenient biomaterial to generate adipose tissues for different applications. To date, gelatin sponge or gelatin hydrogels have been used to create adipose tissues from human MSCs [32, 34, 35]. In these studies, MSCs displayed reduced adipogenic potential in gelatin cultures when compared to monolayer cultures. Therefore, the optimal conditions that can facilitate MSC adipogenesis within scaffolds, such as those formulated from gelatin, still need to be developed.

It has been well demonstrated that the mechanical properties of substrates, including biomaterial scaffolds, play an important role in mediating MSC differentiation, such as adipogenesis. The first study demonstrating this relationship, in which MSCs were cultured on collagencoated polyacrylamide gels with different levels of crosslinking and different stiffness characteristics, showed that the softer substrates that mimicked the native stiffness of adipose tissue $(2 \mathrm{kPa})$ resulted in significantly enhanced adipogenesis, compared to harder surface [17]. 
Similarly, using 3D alginate hydrogel, it was shown that MSCs cultured in a soft scaffold produced more oil droplets and displayed higher expression levels of adipogenic genes than in stiff scaffolds [18]. Currently, it is generally accepted that softer substrates support higher adipogenesis of MSCs regardless of whether they are in $2 \mathrm{D}$ or $3 \mathrm{D}$ cultures [19]. It should also be noted that the dimensionality of the cultures also affects cell morphology. For example, in 2D cultures, cell spread and extend robustly on a stiff surface or stiff substrate, while in $3 \mathrm{D}$ cultures, cells maintain a round shape in stiff scaffolds [21, 36], which was also observed in our study (Fig. 2). These seemingly opposite results raise the question of the importance of cell morphology in guiding MSC differentiation. In a recent review, Zonderland et al. suggested that, on flat 2D substrates, a spread morphology and increased nuclear translocation of YAP are good predictors of MSC lineage commitment such as adipogenic differentiation [37]; however, in 3D culture, these parameters do not have the same predictive power on MSC differentiation. It was further suggested that cell volume might play an important role. For example, large cell volumes benefit adipogenesis. Our findings supported that a spread cell morphology (Fig. 2) along with higher YAP levels (Fig. 4) are good indicators of higher level of adipogenesis in 3D culture as well.

In addition to stiffness, the type and density of ligands present in the extracellular matrix (ECM) also affect the spread of MSCs, a process that requires complicated interactions between receptors on cells and binding ligands in the ECM. For example, the fibronectin tripeptide (RGD) motif is necessary to allow MSCs to spread on a hard surface [38]. However, excessive ECM binding with matrix seems to negatively affect hBMSC adipogenesis on 2D culture. Therefore, to eliminate the influence of different ligand densities, we varied the illumination time for hydrogel photocuring, rather than gelatin concentration, to control the stiffness of photocrosslinkable gelatin [24].

Currently, there is no consensus regarding the functional association and relationship among YAP/TAZ pathway, substrate stiffness, and adipogenesis. Conflicting results have been reported. On 2D culture, increasing YAP activity suppresses adipogenic differentiation [39]. In contrast, inactivation of YAP using small molecule or siRNA promotes adipogenic differentiation [40]. In a study using gelatin scaffolds with stiffness gradients from $8 \mathrm{kPa}$ to $30 \mathrm{kPa}$, activation of YAP was found in the cells encapsulated in low-stiffness regions [36], similar to our findings here. However, reduced adipogenesis was observed, which is not consistent with our findings (Fig. 3). In another study using polyethylene glycolbased scaffolds, MSCs seeded in soft hydrogels had a larger and more extended cell morphology than those in stiff gels [41]. However, it was found that YAP nuclear function impaired adipogenesis. Moreover, cells exposed to adipogenic stimulation within stiff matrices were shown to displayed higher adipogenesis as compared to those in soft hydrogels. To address this inconsistency, Caliari et al. used the same biomaterials and compared MSC behavior on or within the scaffolds, and showed that increased stiffness promoted cell spreading and YAP/TAZ nuclear localization on 2D culture. However, the opposite trend was observed in cells in $3 \mathrm{D}$ culture [21]. Taken together, it is clear that YAP/TAZ signaling is responsible for cell response to stiffness, but the outcome in terms of adipogenesis depends highly on the dimensionality of the culture system.

Interestingly, Khetan et al. demonstrated that the differentiation of MSCs is dictated by the generation of degradation-mediated cellular traction, which was not directly related to cell morphology or matrix mechanics [42]. Specifically, hBMSCs encapsulated within hyaluronic acid hydrogels that permitted cell-mediated degradation exhibited high degrees of adipogenesis. In our study, we have shown that soft hydrogel is more easily degraded by collagenase than its stiff counterpart (Fig. 1b). Of note, a degradable environment is reported to enhance the activity of YAP/TAZ [21], strongly suggesting the importance of matrix degradation in mediating cell behavior in hydrogels.

Although the central role of YAP/TAZ is to dictate MSC response to stiffness, it is not clear how mechanical cues are converted into the biochemical signals that affect this pathway. Previously, we had found that CAV1, a scaffolding protein of cholesterol-rich caveolae lipid rafts in the plasma membrane involved in membrane receptor traffic [43], regulates proliferation and differentiation of hBMSCs [44-46]. We have hypothesized that CAV1 expression may stabilize the differentiated and undifferentiated stem cell phenotype and that changes in CAV1 expression may be required for transition between the two [45]. We thus investigated in this study whether CAV1 also regulates the response of hBMSCs to stiffness. In a recent study investigating fibroblast behavior on soft and hard substrates (2D culture), the activation of YAP in cells cultured on soft substrates was completely abolished when CAV1 was knocked out [23], indicating that CAV1 positively regulates YAP activity. We have obtained the opposite observation here, namely that the suppression of CAV1 results in higher level of YAP (Fig. 7). Our observation is in fact supported by other studies: for example, in the process of mesothelial-to-mesenchymal transition (MMT), the mechanical stretch-activated YAP/TAZ pathway first activates CAV1 expression, then CAV1 conversely suppresses YAP/TAZ [47]. We speculate that the different observations may have come from the 
different culture conditions (2D versus 3D) used and that CAV1 is unlikely to be the only upstream factor regulating YAP activity. In another study, where CAV1 was overexpressed in hBMSCs, adipogenic differentiation was significantly suppressed [48], consistent with our findings (Fig. 6).

There are some limitations in our study. First, similar to other studies investigating cellular responses to stiffness, this study did not fully eliminate the influence of differing nutrient/waste diffusion rates experienced by the soft and hard scaffolds. However, given the relatively small geometric dimensions of the 3D constructs (2-mm height and 5-mm diameter) and consequently likely limited influence on diffusion related to pore size differences between the soft and stiff scaffolds, this is unlikely to be a major contributing factor. Secondly, whole-cell CAV1 analysis was carried out, and subcellular CAV1 distribution was not done. Namely, we have not analyzed the membrane domain distribution of CAV1, such as association with cholesterol-rich membrane rafts [42, 44], which will be investigated in future studies. Thirdly, our mechanistic study has not included signaling pathways other than Hippo, i.e., YAP/TAZ, that are also known to be related to the cellular response to variations in substrate stiffness, including $\beta$-catenin and Rock/Rho pathways [49-51]. Our future mechanistic investigations will include a more in-depth direct comparison of hBMSC behaviors and phenotypes under $2 \mathrm{D}$ and $3 \mathrm{D}$ cultures, as well as the use of hydrogels that have photo-switchable stiffness to examine the "permanence" of substrate stiffness-mediated cellular signaling.

\section{Conclusions}

Our study demonstrated that softer photocrosslinked 3D gelatin scaffolds promoted more cell spreading and enhanced adipogenesis of encapsulated hBMSCs when compared to more stiff hydrogels. However, the proadipogenic effect of the softer scaffold alone was less efficient than that of the adipogenic supplements found in widely used adipogenic culture medium, indicating the importance of biochemical cues. Interestingly, hBMSCs cultured in soft gels displayed reduced levels of CAV1 and increased YAP activity, suggesting CAV1 action upstream of YAP. Given the known involvement of the YAP pathway in mechanosensing of substrate stiffness and its complex downstream signaling pathway, which is also modulated as a function of the dimensionality of the culture system, our findings reported here suggest that suppressing CAV1 expression is likely an efficient way to enhance adipogenesis. The 3D culture models developed here also provide a robust tool to study other behaviors of MSCs in response to stiffness, such as osteogenesis, neurogenesis, and chondrogenesis.

\section{Abbreviations}

hBMSCs: Human bone marrow-derived mesenchymal stem cells; CAV1: Caveolin-1; YAP/TAZ: Yes-associated protein/Tafazzin; siRNA: Small interfering RNA; qRT-PCR: Quantitative real-time polymerase chain reaction; 2D: Two-dimensional; 3D: Three-dimensional; GelMA: Methacrylated gelatin; LAP: Lithium phenyl-2,4,6-trimethyl-benzoyl phosphinate; PBS: Phosphatebuffered saline; PBS-T: Phosphate-buffered saline-Tween;

PFA: Paraformaldehyde; GM: Growth medium; AM: Adipogenic medium; RIPA: Radioimmunoprecipitation assay; PVDF: Polyvinylidene fluoride; HRP: Horseradish peroxidase

\section{Supplementary Information}

The online version contains supplementary material available at https://doi. org/10.1186/s13287-021-02356-z.

\section{Additional file 1: Figure S1. Relative expression levels of adipogenic marker genes in hBMSCs cultured under adipogenic conditions (AM) in soft or stiff scaffolds. Data are normalized to that from Soft-GM group (cultured under control growth conditions). $N=4$. $^{*}, p<0.05 ;{ }^{* *}, p<0.01$; ****, $\mathrm{p}<0.0001$. $\mathrm{GM}=$ growth medium; $\mathrm{AM}=$ adipogenic medium. Figure S2. Relative expression level of CAV1 at $48 \mathrm{~h}$ after transfection. Data are normalized to that in CTRL group, which was not treated with siRNA or transfection agents. $N=4$. ${ }^{* * *}, p<0.001 ;{ }^{* * *}, p<0.0001$. Table S1. Primer sequences for qRT-qPCR. Table S2. Information of antibodies used in this study. \\ Additional file 2. \\ Additional file 3 .}

\section{Acknowledgements}

The authors gratefully acknowledge Dr. Paul Manner (University of Washington) for providing the human tissue samples for hBMSC isolation. We thank Dr. Jian Tan for isolating and expanding hBMSCs. The Center for Biological Imaging (CBI) at the University of Pittsburgh is acknowledged for supporting the confocal imaging. Figure 8 was created using materials from BioRender.com.

\section{Authors' contributions}

SX collected, processed, analyzed and interpreted data, and wrote the manuscript. ZL collected, analyzed and interpreted data, and edited the manuscript. MRF collected data and edited the manuscript. LL collected data and reviewed the manuscript. JS analyzed and interpreted data, and edited the manuscript. NB conceptualized, interpreted data, and edited the manuscript. HL conceptualized and designed experiments, analyzed and interpreted data, wrote the manuscript, and obtained funding. RST conceptualized the study, interpreted data, contributed to manuscript writing and editing, and obtained funding. The authors approved the final manuscript.

\section{Funding}

This work was partially supported by funding from the National Institutes of Health (R01EB019430) and Department of Orthopaedic Surgery at the University of Pittsburgh (to HL).

\section{Availability of data and materials}

The data sets supporting the conclusions of this article are included within the article and its supplementary files.

\section{Declarations}

Ethics approval and consent to participate

The human bone marrow was harvested from patients undergoing total knee arthroplasty with IRB approval by the University of Washington School of Medicine (07-5025-E/A 01) and the University of Pittsburgh Medical Center (PRO14020504) using protocols that exempted consent collection from patients to the surgical waste materials.

Consent for publication

Not applicable. 


\section{Competing interests}

RST is an Editor-in-Chief of Stem Cell Research \& Therapy and was not involved in the peer-review or editorial handling of the manuscript. The authors have no other competing interests to disclose.

\section{Author details}

'Center for Cellular and Molecular Engineering, Department of Orthopaedic Surgery, University of Pittsburgh School of Medicine, Pittsburgh, Pennsylvania, USA. '2Department of Orthopaedics, The Second Xiangya Hospital of Central South University, Changsha, Hunan, China. ${ }^{3}$ Department of Chem/Petroleum Engineering and Mechanical Engineering \& Materials Science, University of Pittsburgh Swanson School of Engineering, Pittsburgh, Pennsylvania, USA. ${ }^{4}$ McGowan Institute for Regenerative Medicine, University of Pittsburgh, Pittsburgh, Pennsylvania, USA. ${ }^{5}$ Present Address: Biogen, Boston, Massachusetts, USA. ${ }^{6}$ Present Address: Department of Oral Biology, University of Pittsburgh School of Dental Medicine, Pittsburgh, Pennsylvania, USA. 'Department of Bioengineering, University of Pittsburgh Swanson School of Engineering, Pittsburgh, Pennsylvania, USA. ${ }^{8}$ Present Address: Institute for Tissue Engineering and Regenerative Medicine, The Chinese University of Hong Kong, Shatin, Hong Kong SAR, China.

\section{Received: 28 March 2021 Accepted: 26 April 2021}

\section{Published online: 14 June 2021}

\section{References}

1. Mahoney CM, Imbarlina C, Yates CC, Marra KG. Current therapeutic strategies for adipose tissue defects/repair using engineered biomaterials and biomolecule formulations. Front Pharmacol. 2018;9:507. https://doi. org/10.3389/fphar.2018.00507.

2. Bellini E, Grieco MP, Raposio E. The science behind autologous fat grafting. Ann Med Surg (Lond). 2017;24:65-73. https://doi.org/10.1016/j.amsu.2017.11. 001

3. Kokai LE, Marra K, Rubin JP. Adipose stem cells: biology and clinical applications for tissue repair and regeneration. Transl Res. 2014;163(4):399408. https://doi.org/10.1016/j.trsl.2013.11.009.

4. Flynn L, Prestwich GD, Semple JL, Woodhouse KA. Adipose tissue engineering with naturally derived scaffolds and adipose-derived stem cells. Biomaterials. 2007;28(26):3834-42. https://doi.org/10.1016/j.biomaterials.2007. 05.002.

5. Tencerova M, Kassem M. The bone marrow-derived stromal cells: commitment and regulation of adipogenesis. Front Endocrinol (Lausanne) 2016;7:127. https://doi.org/10.3389/fendo.2016.00127.

6. Contessi Negrini N, Bonnetier M, Giatsidis G, Orgill DP, Fare S, Marelli B. Tissue-mimicking gelatin scaffolds by alginate sacrificial templates for adipose tissue engineering. Acta Biomaterialia. 2019;87:61-75. https://doi. org/10.1016/j.actbio.2019.01.018.

7. Shanti RM, Janjanin S, Li WJ, Nesti LJ, Mueller MB, Tzeng MB, et al. In vitro adipose tissue engineering using an electrospun nanofibrous scaffold. Ann Plast Surg. 2008;61(5):566-71. https://doi.org/10.1097/SAP.0b013e3181 6d9579.

8. Engler AJ, Sen S, Sweeney HL, Discher DE. Matrix elasticity directs stem cell lineage specification. Cell. 2006;126(4):677-89. https://doi.org/10.1016/j.cell.2 006.06.044.

9. Discher DE, Janmey P, Wang Y-I. Tissue cells feel and respond to the stiffness of their substrate. Science. 2005;310(5751):1139-43. https://doi.org/1 0.1126/science.1116995.

10. Park J, Kim P, Helen W, Engler AJ, Levchenko A, Kim D-H. Control of stem cell fate and function by engineering physical microenvironments. Integr Biol. 2012:4(9):1008-18. https://doi.org/10.1039/c2ib20080e.

11. Yang C, Tibbitt MW, Basta L, Anseth KS. Mechanical memory and dosing influence stem cell fate. Nat Mater. 2014;13(6):645-52. https://doi.org/10.103 8/NMAT3889.

12. Murphy WL, McDevitt TC, Engler AJ. Materials as stem cell regulators. Nat Mater. 2014;13(6):547-57. https://doi.org/10.1038/NMAT3937.

13. Wen JH, Vincent LG, Fuhrmann A, Choi YS, Hribar KC, Taylor-Weiner H, et al. Interplay of matrix stiffness and protein tethering in stem cell differentiation. Nat Mater. 2014;13(10):979-87. https://doi.org/10.1038/NMAT4051.

14. Kurpinski K, Chu J, Hashi C, Li S. Anisotropic mechanosensing by mesenchymal stem cells. Proc Natl Acad Sci U S A. 2006;103(44):16095-100. https://doi.org/10.1073/pnas.0604182103.
15. Kureel SK, Mogha P, Khadpekar A, Kumar V, Joshi R, Das S, et al. Soft substrate maintains proliferative and adipogenic differentiation potential of human mesenchymal stem cells on long-term expansion by delaying senescence. Biol Open. 2019;8(4). https://doi.org/10.1242/bio.039453.

16. Zhang T, Lin S, Shao X, Shi S, Zhang Q, Xue C, et al. Regulating osteogenesis and adipogenesis in adipose-derived stem cells by controlling underlying substrate stiffness. J Cell Physiol. 2018;233(4):3418-28. https://doi. org/10.1002/jcp.26193.

17. Young DA, Choi YS, Engler AJ, Christman KL. Stimulation of adipogenesis of adult adipose-derived stem cells using substrates that mimic the stiffness of adipose tissue. Biomaterials. 2013;34(34):8581-8. https://doi.org/10.1016/j. biomaterials.2013.07.103

18. Chaudhuri O, Gu L, Klumpers D, Darnell M, Bencherif SA, Weaver JC, et al. Hydrogels with tunable stress relaxation regulate stem cell fate and activity. Nat Mater. 2016:15(3):326-34. https://doi.org/10.1038/nmat4489.

19. Hogrebe NJ, Gooch KJ. Direct influence of culture dimensionality on human mesenchymal stem cell differentiation at various matrix stiffnesses using a fibrous self-assembling peptide hydrogel. J Biomed Mater Res A. 2016; 104(9):2356-68. https://doi.org/10.1002/jbm.a.35755.

20. Docheva D, Popov C, Mutschler W, Schieker M. Human mesenchymal stem cells in contact with their environment: surface characteristics and the integrin system. J Cell Mol Med. 2007;11(1):21-38. https://doi.org/10.1111/j.1 582-4934.2007.00001.x

21. Caliari SR, Vega SL, Kwon M, Soulas EM, Burdick JA. Dimensionality and spreading influence MSC YAP/TAZ signaling in hydrogel environments. Biomaterials. 2016; 103:314-23. https://doi.org/10.1016/j.biomaterials.2016.06.061.

22. Yeh YC, Ling JY, Chen WC, Lin HH, Tang MJ. Mechanotransduction of matrix stiffness in regulation of focal adhesion size and number: reciprocal regulation of caveolin-1 and beta1 integrin. Sci Rep. 2017;7(1):15008. https:// doi.org/10.1038/s41598-017-14932-6.

23. Moreno-Vicente R, Pavon DM, Martin-Padura I, Catala-Montoro M, DiezSanchez A, Quilez-Alvarez A, et al. Caveolin-1 modulates mechanotransduction responses to substrate stiffness through actindependent control of YAP. Cell Rep. 2018;25(6):1622-1635 e6. https://doi. org/10.1016/j.celrep.2018.10.024.

24. Lin H, Cheng AW, Alexander PG, Beck AM, Tuan RS. Cartilage tissue engineering application of injectable gelatin hydrogel with in situ visiblelight-activated gelation capability in both air and aqueous solution. Tissue engineering. Part A. 2014;20(17-18):2402-11. https://doi.org/10.1089/ten. TEA.2013.0642.

25. Nichol JW, Koshy ST, Bae H, Hwang CM, Yamanlar S, Khademhosseini A. Cell-laden microengineered gelatin methacrylate hydrogels. Biomaterials. 2010:31(21):5536-44. https://doi.org/10.1016/.biomaterials.2010.03.064.

26. Lin H, Tang Y, Lozito TP, Oyster N, Kang RB, Fritch MR, et al. Projection stereolithographic fabrication of BMP-2 gene-activated matrix for bone tissue engineering. Sci Rep. 2017;7(1):1-11. https://doi.org/10.1038/s41598017-11051-0.

27. Lin Z, Li Z, Li EN, Li X, Del Duke CJ, Shen H, et al. Osteochondral tissue chip derived from iPSCs: modeling OA pathologies and testing drugs. Front Bioeng Biotechnol. 2019;7:411. https://doi.org/10.3389/fbioe.2019.00411.

28. Kraus NA, Ehebauer F, Zapp B, Rudolphi B, Kraus BJ, Kraus D. Quantitative assessment of adipocyte differentiation in cell culture. Adipocyte. 2016;5(4): 351-8. https://doi.org/10.1080/21623945.2016.1240137.

29. Nohata N, Hanazawa T, Kikkawa N, Mutallip M, Fujimura L, Yoshino H, et al. Caveolin-1 mediates tumor cell migration and invasion and its regulation by miR-133a in head and neck squamous cell carcinoma. Int J Oncol. 2011; 38(1):209-17. https://doi.org/10.3892/ijo_00000840.

30. Lin H, Lozito TP, Alexander PG, Gottardi R, Tuan RS. Stem cell-based microphysiological osteochondral system to model tissue response to interleukin-1beta. Mol Pharm. 2014;11(7):2203-12. https://doi.org/10.1021/ mp500136b.

31. Arrigoni C, Lopa S, Candrian C, Moretti M. Organs-on-a-chip as model systems for multifactorial musculoskeletal diseases. Curr Opin Biotechnol. 2020:63:79-88. https://doi.org/10.1016/j.copbio.2019.12.006.

32. O'Donnell BT, Al-Ghadban S, Ives CJ, L'Ecuyer MP, Monjure TA, RomeroLopez M, et al. Adipose tissue-derived stem cells retain their adipocyte differentiation potential in three-dimensional hydrogels and bioreactors. Biomolecules. 2020:10(7):1070. https://doi.org/10.3390/biom10071070.

33. Bello AB, Kim D, Kim D, Park H, Lee $\mathrm{S}-\mathrm{H}$. Engineering and functionalization of gelatin biomaterials: from cell culture to medical applications. Tissue Eng Part B Rev. 2020;26(2):164-80. https://doi.org/10.1089/ten.teb.2019.0256. 
34. Hong L, Peptan I, Clark P, Mao JJ. Ex vivo adipose tissue engineering by human marrow stromal cell seeded gelatin sponge. Ann Biomed Eng. 2005; 33(4):511-7. https://doi.org/10.1007/s10439-005-2510-7.

35. Zou J, Wang W, Neffe AT, Xu X, Li Z, Deng Z, et al. Adipogenic differentiation of human adipose derived mesenchymal stem cells in 3D architectured gelatin based hydrogels (ArcGel). Clin Hemorheol Microcirc. 2017;67(3-4):297-307. https://doi.org/10.3233/CH-179210.

36. Major LG, Holle AW, Young JL, Hepburn MS, Jeong K, Chin IL, et al. Volume adaptation controls stem cell mechanotransduction. ACS Appl Mater Interfaces. 2019;11(49):45520-30. https://doi.org/10.1021/acsami.9b19770.

37. Zonderland J, Moroni L. Steering cell behavior through mechanobiology in 3D: a regenerative medicine perspective. Biomaterials. 2021;268:120572. https://doi.org/10.1016/j.biomaterials.2020.120572.

38. Hogrebe NJ, Reinhardt JW, Tram NK, Debski AC, Agarwal G, Reilly MA, et al. Independent control of matrix adhesiveness and stiffness within a 3D selfassembling peptide hydrogel. Acta Biomaterialia. 2018;70:110-9. https://doi. org/10.1016/.jactbio.2018.01.031.

39. Lorthongpanich C, Thumanu K, Tangkiettrakul K, Jiamvoraphong N, Laowtammathron C, Damkham N, et al. YAP as a key regulator of adipoosteogenic differentiation in human MSCs. Stem Cell Res Ther. 2019;10(1): 402. https://doi.org/10.1186/s13287-019-1494-4.

40. Liu X, Long X, Gao Y, Liu W, Hayashi T, Mizuno K, et al. Type I collagen inhibits adipogenic differentiation via YAP activation in vitro. J Cell Physiol. 2020;235(2):1821-37. https://doi.org/10.1002/jcp.29100.

41. Oliver-De La Cruz J, Nardone G, Vrbsky J, Pompeiano A, Perestrelo AR, Capradossi F, et al. Substrate mechanics controls adipogenesis through YAP phosphorylation by dictating cell spreading. Biomaterials. 2019;205:64-80. https://doi.org/10.1016/j.biomaterials.2019.03.009.

42. Khetan S, Guvendiren M, Legant WR, Cohen DM, Chen CS, Burdick JA Degradation-mediated cellular traction directs stem cell fate in covalently crosslinked three-dimensional hydrogels. Nat Mater. 2013;12(5):458-65. https://doi.org/10.1038/nmat3586.

43. Head BP, Patel HH, Insel PA. Interaction of membrane/lipid rafts with the cytoskeleton: impact on signaling and function: membrane/lipid rafts, mediators of cytoskeletal arrangement and cell signaling. Biochim Biophys Acta. 2014;1838(2):532-45. https://doi.org/10.1016/j.bbamem.2013.07.018.

44. Sohn J, Lin H, Fritch MR, Tuan RS. Influence of cholesterol/caveolin-1/ caveolae homeostasis on membrane properties and substrate adhesion characteristics of adult human mesenchymal stem cells. Stem Cell Res Ther. 2018;9(1):86. https://doi.org/10.1186/s13287-018-0830-4.

45. Baker N, Tuan RS. The less-often-traveled surface of stem cells: caveolin-1 and caveolae in stem cells, tissue repair and regeneration. Stem Cell Res Ther. 2013;4(4):90. https://doi.org/10.1186/scrt276.

46. Baker N, Zhang G, You Y, Tuan RS. Caveolin-1 regulates proliferation and osteogenic differentiation of human mesenchymal stem cells. J Cell Biochem. 2012;113(12):3773-87. https://doi.org/10.1002/jcb.24252.

47. Strippoli R, Sandoval P, Moreno-Vicente R, Rossi L, Battistelli C, Terri M, et al. Caveolin1 and YAP drive mechanically induced mesothelial to mesenchymal transition and fibrosis. Cell Death Dis. 2020;11(8):647. https:// doi.org/10.1038/s41419-020-02822-1.

48. Park JS, Kim HY, Kim HW, Chae GN, Oh HT, Park JY, et al. Increased caveolin1, a cause for the declined adipogenic potential of senescent human mesenchymal stem cells. Mech Ageing Dev. 2005;126(5):551-9. https://doi. org/10.1016/.j.mad.2004.11.014.

49. Janmey PA, Wells RG, Assoian RK, McCulloch CA. From tissue mechanics to transcription factors. Differentiation. 2013;86(3):112-20. https://doi.org/10.1 016/j.diff.2013.07.004

50. Dasgupta I, McCollum D. Control of cellular responses to mechanical cues through YAP/TAZ regulation. J Biol Chem. 2019;294(46):17693-706. https:// doi.org/10.1074/jbc.REV1 19.007963.

51. Virdi JK, Pethe P. Biomaterials regulate mechanosensors YAP/TAZ in stem cell growth and differentiation. J Tissue Eng Regen Med. 2020;18(2):1-17. https://doi.org/10.1007/s13770-020-00301-4.

\section{Publisher's Note}

Springer Nature remains neutral with regard to jurisdictional claims in published maps and institutional affiliations.

Ready to submit your research? Choose BMC and benefit from:

- fast, convenient online submission

- thorough peer review by experienced researchers in your field

- rapid publication on acceptance

- support for research data, including large and complex data types

- gold Open Access which fosters wider collaboration and increased citations

- maximum visibility for your research: over $100 \mathrm{M}$ website views per year

At $\mathrm{BMC}$, research is always in progress.

Learn more biomedcentral.com/submissions 